\title{
STRONG, VARIABLE CIRCULAR POLARIZATION IN PKS 1519-273
}

\author{
JEAN-PIERRE MACQUART \\ RCfTA, School of Physics, University of Sydney, Australia \\ E-mail:jpm@physics.usyd.edu.au \\ LUCYNA KEDZIORA-CHUDCZER \\ Australia Telescope National Facility/Anglo-Australian Observatory, Australia \\ E-mail: lkedzior@atnf.csiro.au \\ DAVID JAUNCEY \\ Australia Telescope National Facility, CSIRO, Australia \\ E-mail: djauncey@atnf.csiro.au \\ DAVID RAYNER \\ Australia Telescope National Facility/University of Tasmania, Australia \\ E-mail:d_rayner@postoffice.utas.edu.au
}

\begin{abstract}
We find strong ( $>1 \%$ ) circular polarization in the intraday-variable radio source PKS 1519-273. The source exhibits $\sim 12$ hourly variability in all four Stokes parameters at 4.8 and $8.6 \mathrm{GHz}$, and longer timescale variability at 2.5 and $1.4 \mathrm{GHz}$. The characteristics and frequency dependence of the variability suggest that it is due to interstellar scintillation. VSOP limits on the distance to the scattering screen constrain the brightness temperature to $T_{B}>5 \times 10^{13} \mathrm{~K}$. The fluctuations in total intensity are well-correlated with those in circular polarization, implying that the variable component of the source is $-3.8 \pm 0.4 \%$ circularly polarized at $4.8 \mathrm{GHz}$. The origin of the circular polarization is unclear.
\end{abstract}

\section{Introduction}

Linear polarization observed in extragalactic radio sources is accepted as a characteristic signature of synchrotron emission. However, the characteristics and interpretation of the circular polarization $(\mathrm{CP})$ observed in some extragalactic sources is unclear; the CP is typically weak and has been difficult to measure reliably. However, the design of the Australia Telescope Compact Array (ATCA) makes it capable of high-accuracy circular polarimetry. Here we present ATCA polarimetric data for the intra-day variable (IDV) source, PKS 1519-273.

The BL-Lac PKS 1519-273 was discovered to exhibit intraday variability in the ATCA IDV Survey (Kedziora-Chudczer et al., 2000). It is identified with a $z>0.4, m_{V}=18.5$ star-like object with a featureless optical spectrum. PKS 1519-273 exhibits persistent variability: it has been observed to vary each of the 7 times it has been observed with ATCA. This is unlike some other IDVs which 


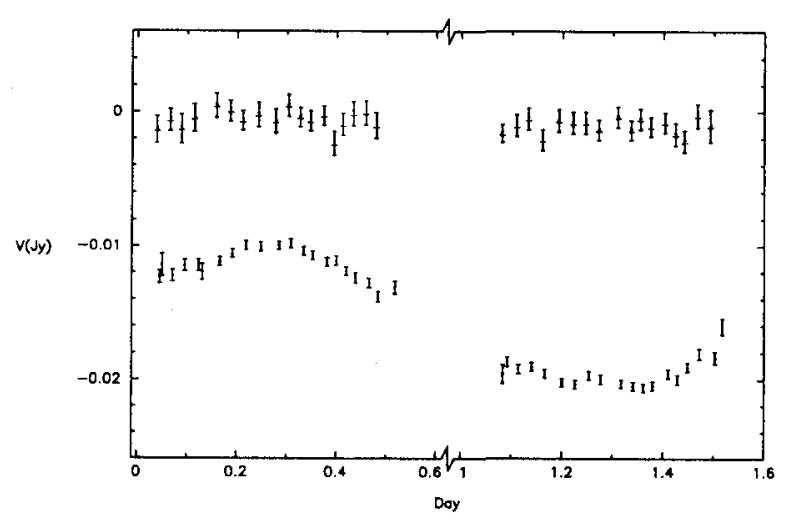

Figure 1. To demonstrate that the variability in $V$ is not instrumental, the $\mathrm{CP}$ at $4.8 \mathrm{GHz}$ in the secondary calibrator PKS 1514-241 (triangles) over a two day interval is plotted with that in PKS $1519-273$.

exhibit episodic scintillation, such as PKS 0405-385 (see Kedziora-Chudczer et al. and Jauncey et al., these proceedings).

\section{Results}

PKS 1519-273 was monitored by ATCA at bands centred on 1.384, 2.496, 4.800 and $8.640 \mathrm{GHz}(128 \mathrm{MHz}$ bandwith) for 5 days. The results at 4.8 and $8.6 \mathrm{GHz}$ are shown in Figure 2. The fluctuations at 1.4 and $2.5 \mathrm{GHz}$ exhibit a longer timescale of variability $(\sim 4$ days at $1.4 \mathrm{GHz})$ and a smaller modulation index in the total intensity.

All four Stokes parameters are observed to vary at all four frequencies, with the exception of Stokes $V$ at $1.4 \mathrm{GHz}$, where instrumental sensitivity renders the detection of the variability marginal. We have eliminated instrumental effects as a possible cause of the variability in the CP (see Figure 1).

The high degree of correlation between the fluctuations in $I$ and $V$ (see Figure 2) indicates an identical origin for their variability. The degree of $\mathrm{CP}$ of the variable component is then $-2.6 \pm 0.5 \%,-3.8 \pm 0.4 \%$ and $-2.4 \pm 1.3 \%$ at 8.6 , 4.8 and $2.5 \mathrm{GHz}$ respectively.

\section{Discussion}

\subsection{Scintillation}

We argue that the variability observed in PKS 1519-273 is due to interstellar scintillation (ISS). If intrinsic to the source the variability timescale implies an angular diameter sufficiently small that the source must exhibit ISS. This interpretation is 

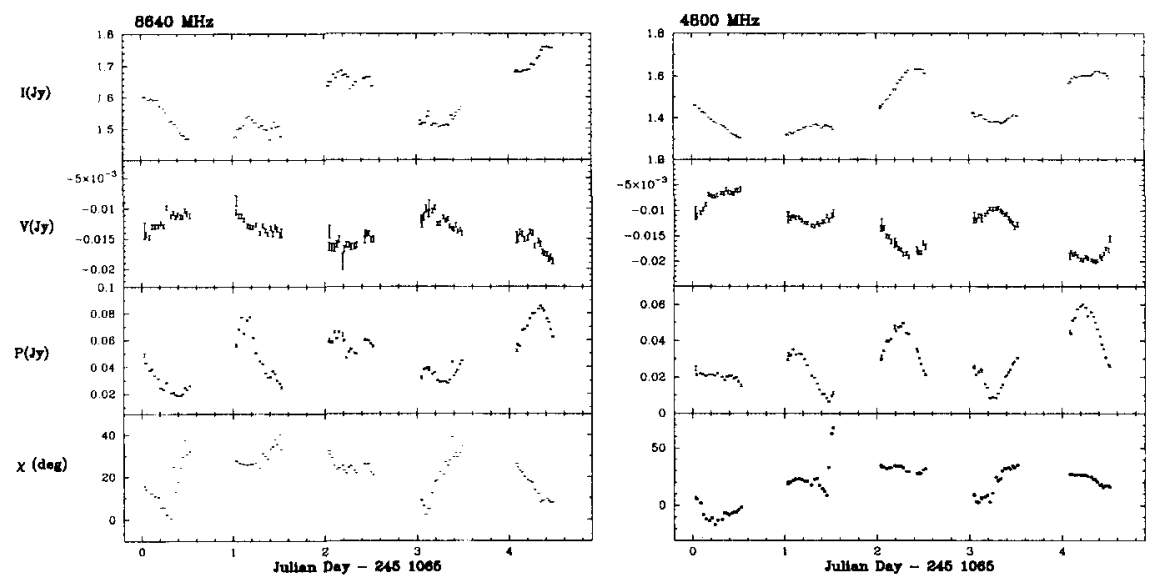

Figure 2. Variability of PKS $1519-273$ at 4.80 and $8.64 \mathrm{GHz}$. Each point represents a 10 min average, and is plotted with $1 \sigma$ error bars.

supported by the longer variability timescales observed at 1.4 and $2.5 \mathrm{GHz}$, consistent with refractive scintillation, with the 4.8 and $8.6 \mathrm{GHz}$ variability explained in terms of weak scattering.

The limit on the angular size set by VSOP at $1.7 \mathrm{GHz}^{\star}$ contrains the distance of the scattering screen to $D>390 \mathrm{pc}$ for a scintillation speed $v>50 \mathrm{~km} \mathrm{~s}^{-1}$. Using the $4.8 \mathrm{GHz}$ data and assuming the results of weak scattering theory, this contrains the angular size to $\theta_{S} \lesssim 37 \mu$ as and the brightness temperature to $T_{B}>5 \times 10^{13} \mathrm{~K}$ at this frequency. If, however, all the $\mathrm{CP}$ observed in this source is associated with the variable component, one obtains a direct measurement of the flux density of the source component participating in the scintillation. One therefore obtains an additional constraint on the scintillation parameters, indicating $T_{B} \approx 6 \times 10^{14} \mathrm{~K}$ for $v=50 \mathrm{~km} \mathrm{~s}^{-1}$ (see Macquart et al., 2000 for more detail).

\subsection{Circular POlarization}

The characteristics and degree of CP detected in the variable component of PKS 1519-273 are difficult to reconcile with theory. The high fractional CP observed in the variable component decreases sharply between 4.8 and $1.4 \mathrm{GHz}$, in disagreement with the $v^{-1 / 2}$ dependence expected from synchrotron theory (Legg and Westfold, 1968).

CP may arise as a result of conversion from linear polarization via circular repolarization in a relativistic pair plasma (Pacholczyk, 1973; Jones and O'Dell, 1977). In such a medium the natural wave modes are elliptical (or nearly linear for a pair-dominated plasma), and the birefringence of the medium causes linear polarization to be converted to $\mathrm{CP}$ and back in a cyclic fashion, analogous to the

* See http://www.vsop.isas.ac.jp/general/pr/1519-273.gif 
effect of Faraday rotation which converts linear polarization between Stokes $Q$ and $U$. Such conversion is characterized by a strong frequency dependence on the sign of the CP. However, we detect no changes in the sign of the CP between 8.6 and 1.4 GHz. Inhomogeneity and optical depth effects in a pair plasma (Jones and O'Dell, 1977) may account for the observed properties of the CP, but only if the compact component of the source becomes optically thick at $5 \pm 1 \mathrm{GHz}$, near the frequency at which high CP is observed.

\section{Conclusion}

The variability observed in PKS $1519-273$ is well-explained by scintillation theory. However, the high brightness temperature $T_{B}>5 \times 10^{13} \mathrm{~K}$ - possibly as high as $T_{B} \approx 6 \times 10^{14} \mathrm{~K}$ - and the exceptionally high $\mathrm{CP}$ observed in this source are difficult to account for. These two problems may be related, particularly if the high brightness temperature requires that we abandon the hypothesis that the source's emission is due to synchrotron radiation. However, it also is possible that the CP is induced by scintillation itself, in association with fluctuations in the rotation measure (Macquart and Melrose, 2000a,b).

\section{Acknowledgements}

The Australia Telescope is funded by the Commonwealth Government for operation as a national facility by the CSIRO.

\section{References}

Jones, T.W. and O'Dell, S.L.: 1977, Transfer of radiation in self-absorbed synchrotron sources, Astrophys. J. 215, 236.

Kedziora-Chudczer et al:: 2000, The ATCA intraday variability survey of extragalactic sources, Mon. Not. R. Astron. Soc., submitted.

Legg, M.P.C. and Westfold, K.C.: 1968, Elliptic polarization of synchrotron radiation, Astrophys. J. $154,99$.

Macquart, J.-P., Kedziora-Chudczer, L., Jauncey, D.L. and Rayner, D.P.: 2000, Strong, variable circular polarization in PKS 1519-273, to appear in Astrophys. J. 538.

Macquart, J.-P. and Melrose, D.B.: 2000a, Circular polarization induced by scintillation in a magnetized medium, Phys. Rev. E, in press.

Macquart, J.-P. and Melrose, D.B.: 2000b, Scintillation-induced circular polarization in pulsars and quasars, Astrophys. J., submitted.

Pacholczyk, A.G.: 1973, Circular repolarization in compact radio sources, Mon. Not. R. Astron. Soc: $163,29 \mathrm{P}$. 